2 Caffeine as nitrogen source for Reduce Graphene

3 doping, and its functionalization with silver

\title{
4 nanowires
}

\author{
D. Ramírez-González ${ }^{1,7}$, J. de J. Cruz-Rivera ${ }^{2}$, H. Tiznado ${ }^{3}$, A.G. Rodríguez ${ }^{4}$, I. \\ Guillén-Escamilla ${ }^{5}$ and A. Zamudio-Ojeda ${ }^{6, *}$ \\ 1 Posgrado Fis. Mat. CU Valles, Universidad de Guadalajara, México; danielglz@gmx.com \\ 2 Instituto de Metalurgia, Universidad Autónoma de San Luis Potosí, México; jdjcr35@uaslp.mx \\ $3 \mathrm{CNyN}$, Universidad Nacional Autónoma de México, México; tiznado@cnyn.unam.mx \\ 4 CYACYT-CARIEM, Universidad Autónoma de San Luis Potosí, México; angel.rodriguez@uaslp.mx \\ 5 D. Ciencias Naturales y Exactas, CU Valles, Universidad de Guadalajara, México; iguillen1978@gmail.com \\ 6 D. Física, CUCEI, Universidad de Guadalajara, México; nanozam@gmail.com \\ ID-NANO México; danielglz@gmx.com \\ * Correspondence: nanozam@gmail.com; Tel.: +52 (33) 13785900
}

\begin{abstract}
In this work, we propose an easy and a low cost method for the synthesis of Nitrogen-Doped Graphene NDG and its silver nanowires NW functionalization NWGN. The synthesis was performed using the improved graphene oxide method, chemical reduction of graphene oxide in the presence of caffeine as green nitrogen source and the subsequently the silver nanowires growth in the surface, by the chemical reductions salts in the presence of NG. Achieving a homogeneous growing (coating) of graphene sheets. The samples were analyzed using conventional characterization techniques: SEM-EDX, XRD, FT-IR, RAMAN, TEM, HRTEM, STEM and XPS.
\end{abstract}

Keywords: Caffeine, nitrogen-doping, graphene, nanowires, functionalization, pyridinic, monolayer, synthesis, carbon nanostructures, nanomaterials.

\section{Introduction}

Graphene is a two-dimensional material composed only of carbon atoms, which are packed in a honeycomb lattice covalently bonded. Since the report of the synthesis of graphene in 2004, developed for A. Geim and K. Novoselov [1] , the scientific community has developed a series of study about the fascinating properties of this material: high electrical conductivity [2,3], quantum hall effect at room temperatures [4,5-7], high surface area [8,9], charge transfer [11,12], atomic interactions with molecules [12-14], high thermal conductivity [15-17], high transmittance [18,19]. Besides, the properties of Graphene can be modified by the inclusion in their carbon bidimensional structure, foreign atoms as nitrogen [20-22], phosphorous and boron [23,24], or hetero-doping [25]; in this way, there are known three doping types for this: i) pyridinic doping [26], ii) graphitic or substitutional doping [27] and iii) pyrrolic doping [27]. Each one has a certain quantity of atoms that can be included in the carbon hexagonal network. The final properties obtained in the doped Graphene, depends of the kind of atom [28,29]; in the present work, we have selected the nitrogen atom, in order to promote areas with reactivity [26,30,31]; the main objective is the $\mathrm{N}$ insertion in the hexagonal network by removing a carbon atom during the thermally reduction process in presence of the nitrogen source; this atom insertion induces structural defects in the honeycomb lattice, this doping, in a certain percentage concentration, it decreases the crystallinity of the material, and subsequently, changes in the graphene intrinsic properties [32,33]. 
The NDG has different physical-chemical properties in comparison with intrinsic graphene; due to the charge distribution is influenced by the nitrogen dopant and the doping types. Furthermore insertion of foreign atom in the 2D network, favors the generation of activated sites for to promote cluster nucleation, growth nanostructures and anchoring different elements or molecules, in order to produce hybrid or functional materials [22,32,34]. For the synthesis, there are two main routes to fabricate doped Graphene: Direct Doping Synthesis or bottom-up as CVD (chemical vapor deposition) that involves high temperatures, hydrocarbon source, inert atmosphere, high quality copper substrates [20]; MBE (molecular beam epitaxial), this method involves ultra high vacuum, hydrocarbon source, the yield of product is very poor; Plasma, that involves strong magnetic fields for ion implantation; it is important to note that these methods need special and costly infrastructure. The other methods known are Doping Post Treatment or top-down, the most used are: Arc-discharge, that employ two electrodes, controlled atmosphere and high currents; ball milling, that employs high energy to grind up graphite and mix with the doping source in order to promote the atom bonding in the defects; and the doping by hydrothermal methods or wet chemical, with this, we can obtain high yield, it involves autoclave reactors and in most of cases, dangerous doping sources [20,35]. In our case, we use a combination of wet chemical methods and low temperature for heat treatment, a green nitrogen source (caffeine) Figure 1, in order to GOx impregnation via hydrothermal method, then a relative low temperature $\left(600^{\circ} \mathrm{C}\right)$; the heat treatment was conducted in a reductive atmosphere (Ar-H 50\%). Another goal is the fact that we have grown silver nanowires on the surface NDG; most of Research Groups functionalize carbon nanostructures with spherical shape nanoparticles. The functionalization experiments were carried out in an ethylene glycol reflux and silver salts, using the seedless method [37] in presence of NDG sheets.

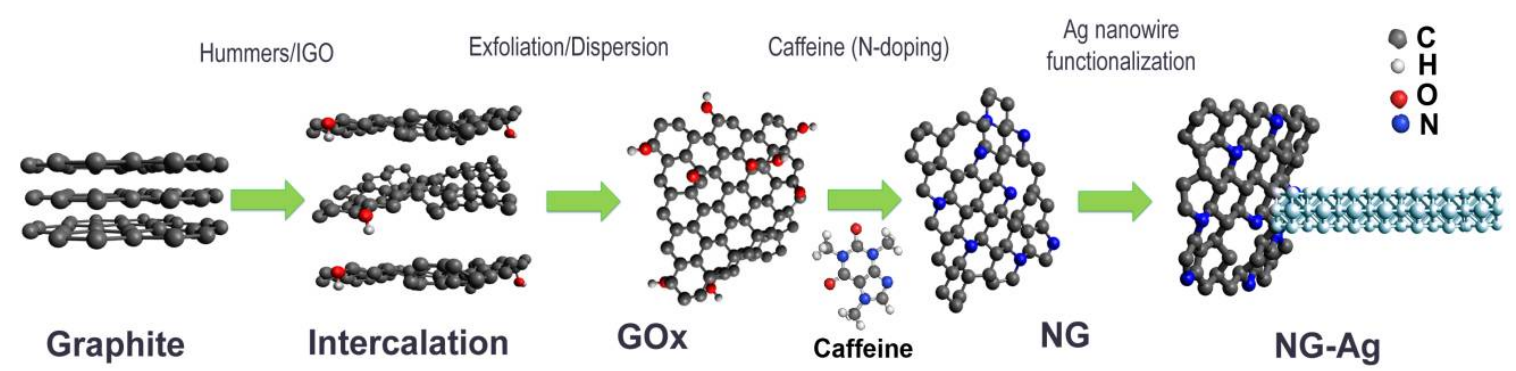

Figure 1. Graphical representation for the experimental process: Graphite to NWNDG.

\section{Materials and Methods}

Graphene Oxide (GOx), partially reduced Graphene (RG) impregnated with caffeine, Nitrogen Doped Graphene (NG) and Silver nanowires functionalized Nitrogen doped Graphene (NWNDG) were sintetized as following. Materials: the materials used in this study, were purchased from Sigma Aldrich, reagent grade, and used as is: graphite flakes CAS 7782-42-5, $\mathrm{H}_{2} \mathrm{SO}_{4}$ CAS 7664-93-9, $\mathrm{H}_{3} \mathrm{PO}_{4}$ CAS 7664-38-2, $\mathrm{KMnO}_{4}$ CAS 7722-64-7, $\mathrm{AgNO}_{3}$ CAS 7761-88-8, $\mathrm{C}_{8} \mathrm{H}_{10} \mathrm{~N}_{4} \mathrm{O}_{2}$ CAS 58-08-2, PVP wt 30k CAS 9003-39-8, 30\% $\mathrm{H}_{2} \mathrm{O}_{2}$ and distilled water. GOx Synthesis: for the synthesis of Graphene Oxide, we carried out as described Marcano et al [36]. Our first stage product was slurry like, dark brown at $3 \%-6 \%$ in solids, hydrophilic. Caffeine GOx impregnation and Chemically partial reduction: hydrothermal process, the experiment was carried out in order to dissolve 1,3,7 trimethylxanthine (caffeine) and impregnated the entire GOx surface, as follow: 150mg of GOx was added in flask ball with $150 \mathrm{ml}$ distilled $\mathrm{H}_{2} \mathrm{O}$, the solution was mixed at $600 \mathrm{rpm} 30 \mathrm{~min}$ and ultrasonic dispersion $(40 \mathrm{kHz} / 150 \mathrm{~W})$ was applied at the same time, in order to keep an homogeneous amber colloidal solution; in a hot stirrer plate, the temperature was increased to $90^{\circ} \mathrm{C}$ using at 300-400 rpm; Then was added into solution in the followed order: caffeine $15 \mathrm{mg}$, ethylene glycol $15 \mathrm{ml}$, and agent reductor $\mathrm{NH}_{4} \mathrm{OH} 15 \mathrm{ml}$; the temperature was keeping at $90 \mathrm{~min}$ in a reflux mode, finally the solution turn black. After the impregnation processes with caffeine, the partially reduced RG or 
chemically reduced Graphene was filtered and dried in oven $70-80^{\circ} \mathrm{C}$ during 4 hours. Nitrogen doping: NDG was obtained as following: the RG impregnate with caffeine was annealed at $650^{\circ} \mathrm{C}$ by $30 \mathrm{~min}$, under reductive atmosphere composed by a mix of gases in equal proportions $\mathrm{Ar} / \mathrm{H} 50-50$, into a quartz tube reactor. NDG Silver NW functionalization NWNDG: In a three necked flask, $150 \mathrm{mg}$ NDG was added to $150 \mathrm{ml}$ ethylene glycol in a reflux setup, the solution at $150^{\circ} \mathrm{C}$ and at 800-1000 rpm; after, simultaneously $50 \mathrm{ml}$ ethylene glycol solutions PVP with concentration $0.6 \mathrm{M}$ and $\mathrm{AgNO}_{3}$ with a concentration $0.1 \mathrm{M}$ were dropping in the main solution and keeping refluxing and mixing by $180 \mathrm{~min}$. The final product was filtered and dried in a convection oven $90^{\circ} \mathrm{C} 3$ hours. Characterization: the characterization was carried out with the following techniques: X-Ray Diffraction (XRD), measurements in Bruker D8 Advance diffractometer. The vibrational characteristics of the Graphenes were analyzed in a HORIBA Xplora Plus Raman spectrometer using $0.5 \mathrm{~mW}-1 \mathrm{~mW} 785 \mathrm{~nm}$ laser. Morphological and chemical composition characteristics were determined with the following electron microscopes: environmental scanning electron microscope E-SEM JEOL 6610LV equipped with EDS Oxford. Transmission electron microscopes: TEM JEOL 100CX 100kV, HRTEM/STEM JEOL 2100 200kv with EDS Oxford, FEG HRTEM FEI Tecnai G2 20 STWIN $300 \mathrm{kV}$ and EDS EDAX. Functional groups were determined using Thermo Scientific i550 Nicolet ATR/FTIR spectrometer for powders. X-ray photoelectron spectroscopy XPS, was used to analyze the type of the nitrogen doped graphene.

\section{Results and discussion}

\subsection{Results and discussion of each stage (experimental matrix)}

\subsubsection{GOx Morphology}

The pristine or initial material were graphite flakes and according to the SEM analysis, each one has $1 \mathrm{~mm}$ lateral size or square and $20 \mu \mathrm{m}$ of thickness in Figure $2 \mathrm{a}$ and Figure $2 \mathrm{~b}$, that is equals to 60,000 stacked sheets of single layers; the SEM micrographs reveals that the morphology obtained for GOx is composed by large single layers, according to the scale bar, they are up $500 \mu \mathrm{m}$ Figure 2c; TEM micrographs confirm that GOx is composed by continuous single layers, Figure $2 \mathrm{~d}$.
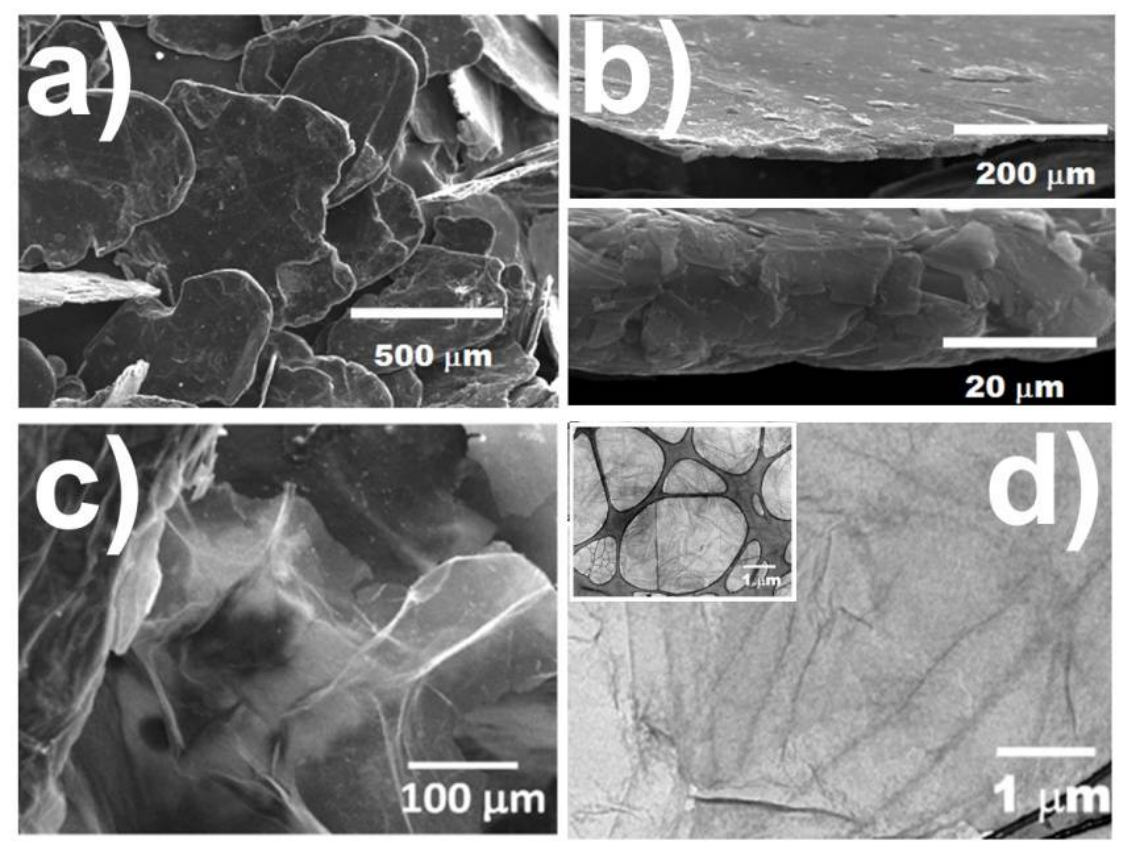

Figure 2. a) SEM Graphite flakes, b) SEM individual graphite flake. c) SEM-GOx, d) TEM-GOx, inset lower 
XRD analysis Figure 3a shows a comparison between graphite and GOx, for graphite, the main peak of intensity at $2 \theta=26.1^{\circ}$ corresponds to the graphite planes [002] with an interlayer distance $\mathrm{d}=0.34 \mathrm{~nm}$, meanwhile for GOx, the main intensity peak is near $2 \theta=10^{\circ}$, that corresponds to interlayer distance $d=1.2 \mathrm{~nm}$, this is due the oxygen and functional groups intercalation $(\mathrm{OH}$ and $\mathrm{COOH})$ in graphite planes as a result of the treatments with strong acid agents; the RAMAN spectra Figure 3b, compares graphite and GOx resulted from the experiment; for graphite (see the inset: free-defects model) the spectrum shows the typical G band at $1574 \mathrm{~cm}^{-1}$, it is first order vibration of the layers meanwhile for GOx (see the inset: model with defects, oxygen and functional groups), the spectrum shows two characteristic peaks: the band $G$ at $1597 \mathrm{~cm}^{-1}$ and the band $\mathrm{D}$ at $\mathrm{cm}^{-1}$, that confirm the change in the surface of basal planes due the oxidation and functional groups attached; as is showing in the FTIR spectra in Figure 3c, there are the following functional groups in the GOx: C-O at $1037 \mathrm{~cm}-1, \mathrm{OH}$ at $1308 \mathrm{~cm}^{-1}, \mathrm{C}=\mathrm{C}$ at $1621 \mathrm{~cm}^{-1}, \mathrm{OH}$ at $1673 \mathrm{~cm}^{-1}, \mathrm{COOH}$ at $2518 \mathrm{~cm}^{-1}$ and all of them promote changes in the interlayer distance that results in a facile route for exfoliation and to obtain GOx single layers, due the decreasing of attraction forces (energy) between neighbors planes. HRTEM micrographs Figure 3d, shows a large single layer GOx.
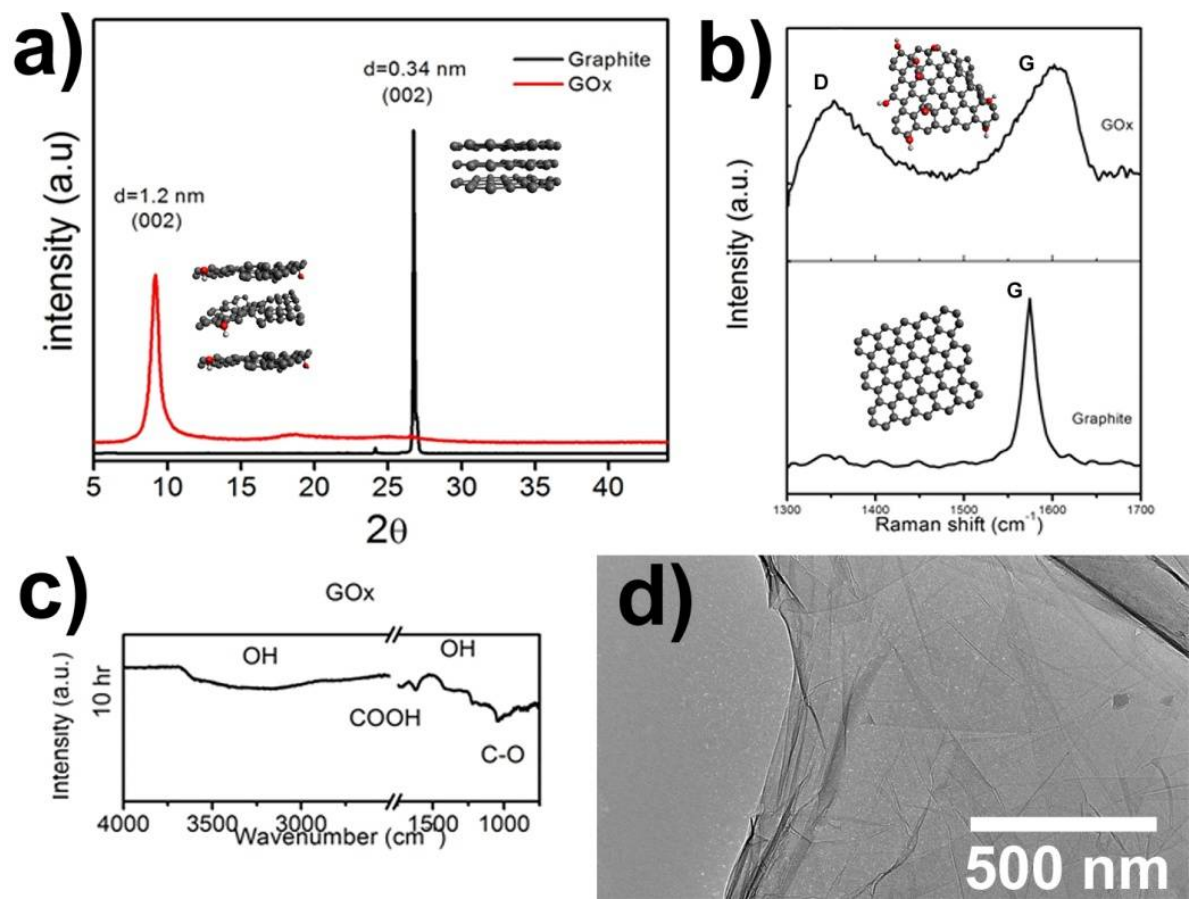

Figure 3. a) XRD of Graphite and GOx showing the increase of interlayer distance in plane (002), from $0.34 \mathrm{~nm}$ to $1.2 \mathrm{~nm}$; b) RAMAN spectra for Graphite and GOx showing D band and G after intercalation; c) FTIR shows $\mathrm{OH}$ and $\mathrm{COOH}$ functional groups presents in GOx; d) TEM of GOx.

\subsubsection{Nitrogen doped Graphene NDG analysis.}

The NDG morphology analyzed by SEM Figure 4a, shows large films, free from agglomerates, there is no other particles or crystals over at the surface, there is no dramatically surface changes, it means that the only change is in the atomic structure, intercalation of nitrogen atoms. In HRTEM analyze, the structure presents the same morphology to GOx, composed by thin layers; according to the EDX chemical analyze, the nitrogen detected in the sample is $9.3 \%$ in weight Figure $4 b$, this indicate that we have a large percent of doping using caffeine. 


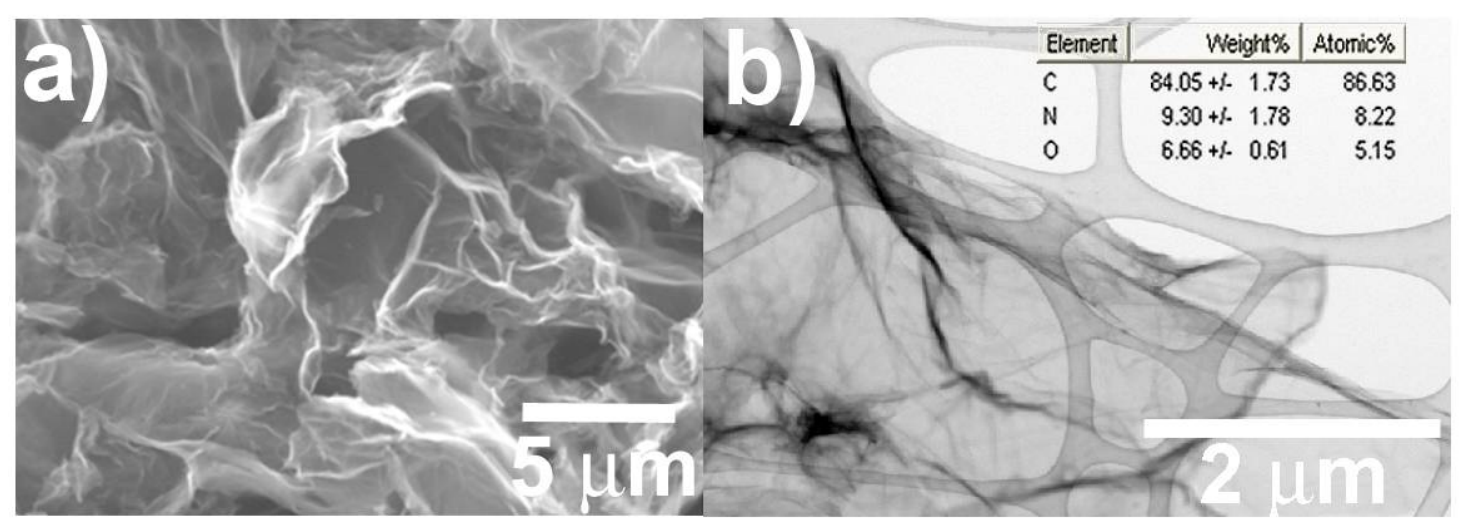

Figure 4. a) SEM NDG; b) HRTEM and EDX quantification of NDG with a content of nitrogen: 9.3\% wt.

At this point, it is important to mention that this method involves an easy experimental set-up and a friendly-green nitrogen source, that according to CN Rao et.al [38]. There are several methods to produce nitrogen doped Graphene that involves expensive experimental setup as CVD, ball milling, plasma enhanced CVD, Arc-discharge, dangerous chemicals, reagents and gases like ammonium or large periods of synthesis and high temperature. In order to explore the average distribution of nitrogen in the obtained doped graphene sample, we use high resolution $200 \mathrm{kV}$ STEM bright field in an isolated single layer Figure 5a, in an EDX line scan analysis Figure 5b, carbon (red line) and nitrogen (green line) are presented in a normalized graph, both signals are in the same path that indicates the homogeneous distribution of both elements, meanwhile an EDX surface elemental mappings are presented in an individual frames for each element: carbon distribution in color red Figure $5 \mathrm{c}$ is present in the graphene zone and in the grid formvar specimen support, and nitrogen distribution in color aqua-blue Figure $5 d$, is just present in the graphene region, note that it is not present in the specimen support.
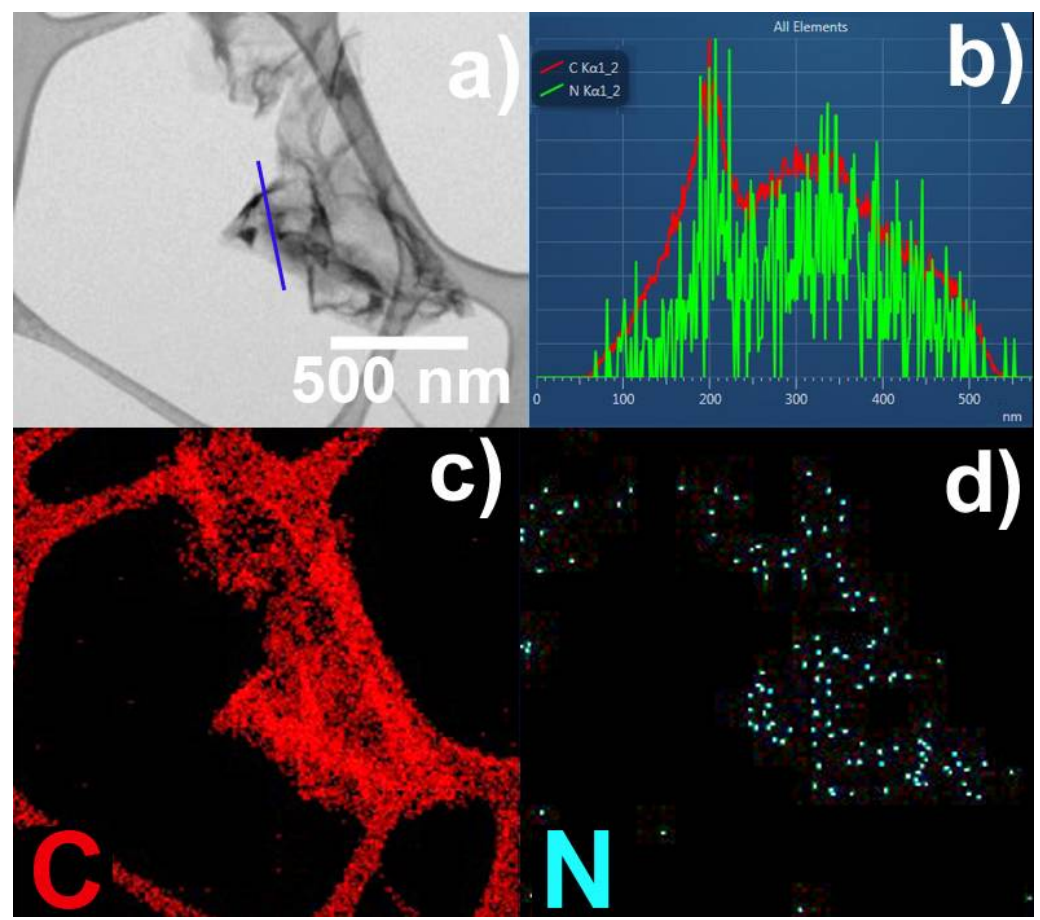

Figure 5. (a) STEM bright field of NDG; (b) normalized Elemental EDX line scan of NG: carbon is colored red, Nitrogen is colored green; (c) y (d) elemental EDX mapping of NG: carbon distribution is colored red, meanwhile nitrogen distribution is colored blue-aqua.

Regarding the nature or type of nitrogen doping, the binding energy between neighbor atoms, is unique for each material, and the properties can change if we modify experimentally this binding, in this case, we analyze via high resolution X-ray photoelectron spectroscopy (XPS), the result binding in each stage was shown, we divide the spectra in region of energy to analyze the type of 
166

167

168

169

170

171

172

173

174

175

176

177

178

179

180

181

182

183

184

185

186

187

188

189

190

191

192

193

194

each one, we analyze and compare the results with a database XPS reference and other authors; the main spectra shows peaks around $284 \mathrm{eV}$ that are corresponding to the binding energy $\mathrm{C}-\mathrm{C}\left(\mathrm{sp}^{2}\right)$ at $284.5 \mathrm{eV}$ for all carbon samples (graphite, RG and GOx) additionally for GOx, the spectra shows a wide peak around $286 \mathrm{eV}$, that corresponds to bindings C-OH/C-O-C at $(286.3,286.7) \mathrm{eV}$ respectively and around $288 \mathrm{eV}$ the bindings $\mathrm{C}=\mathrm{O} / \mathrm{O}-\mathrm{C}=\mathrm{O}(288,289) \mathrm{eV}$ respectively Figure $6 \mathrm{a}$, the binding $\mathrm{C}-\mathrm{O}$ is present only in GOx sample around $532 \mathrm{eV}$ : exactly at $531.5-532 \mathrm{eV}$ as shown in Figure $6 \mathrm{~b}$; the goal of nitrogen doping and its type was found in the XPS spectra around $400 \mathrm{eV}$ : the two main peaks of energy corresponds to pyridine doping type (GN-p) at $398.9 \mathrm{eV}$ and quaternary or substitutional doping type (GN-q) at $400.6 \mathrm{eV}$ Figure $6 \mathrm{c}$, this results are consistently to the results of other groups $[20,30,32,33,39]$. In Figure $6 \mathrm{~d}$ are the models of the obtained system in each stage: graphite is the pristine system, GOx is the result of acid treatment for oxidation and functional groups intercalation, the reduce graphene RG and NDG with the use of caffeine as source of nitrogen.

a)

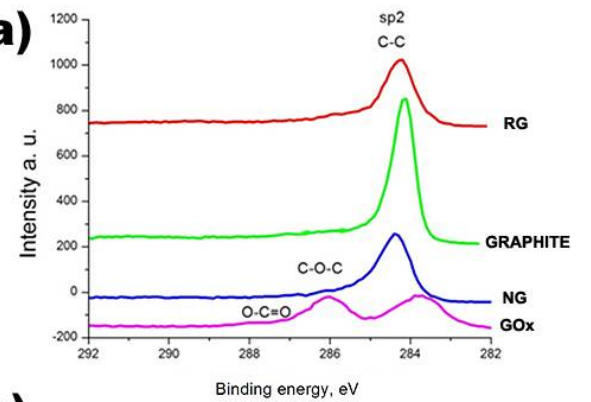

b)

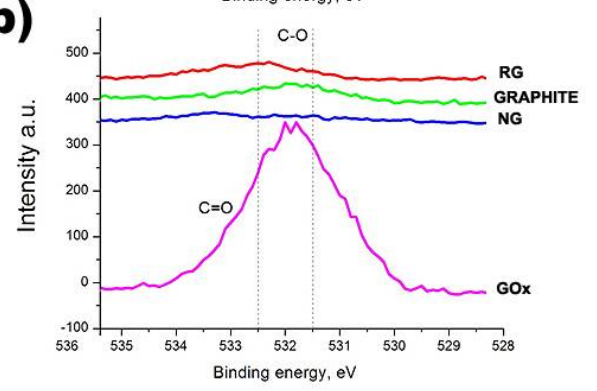

c)

d)
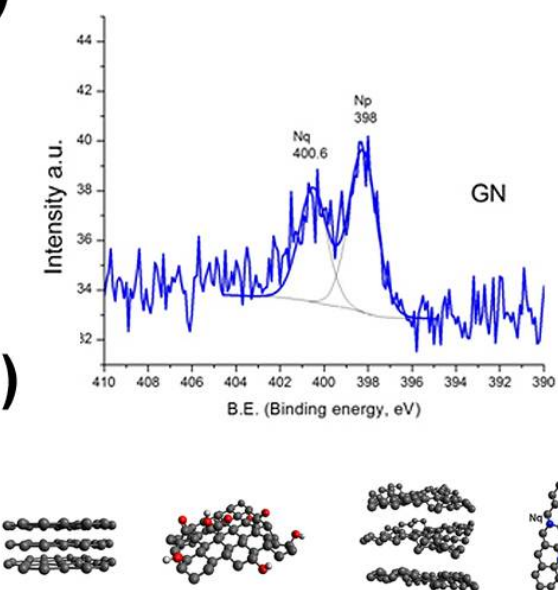

Graphite
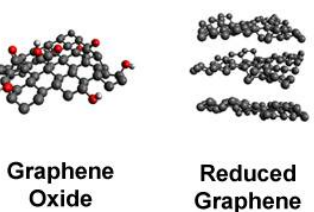

Reduced Reduced
Graphene

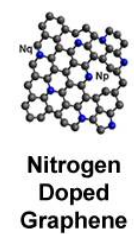

Figure 6. (a) XPS binding energy for C-C sp² around $284 \mathrm{eV}$ for NDG, graphite, RG, meanwhile for GOx, the bonding is drift to the right; (b) C-O bonding only is present in GOx; (c) XPS in the range $390 \mathrm{eV}$ to $410 \mathrm{eV}$ shows two peaks, that correspond to doping quaternary or substitutional in $400.6 \mathrm{eV}$ and pyridinic at $398 \mathrm{eV}$;(d) models for Graphite, GOx, RG and NDG.

\subsubsection{Silver NW Functionalization}

After nitrogen doping, the silver nanowires functionalization on Graphene was analyzed via SEM, HRTEM-STEM/EDX mappings, in order to see the shape and distribution of Ag nanoparticles on the entire NDG sheet. It is important to mention that we made a previous silver nanoparticles functionalization experiment with two types of Graphenes, both at the same conditions: the first one was RG (reduced Graphene) and the second one NDG (Nitrogen Doped Graphene), in order to analyze and compare the surface reactivity of the NDG; the results were characterized with TEM, we found that the NDG promotes more Ag nanoparticles nucleation, because its reactivity, it means that the nitrogen atoms act as active sites on the graphene surface; according to the TEM micrographs Figure $7 \mathrm{a}$ and Figure $7 \mathrm{~b}$, most of the NDG surface were covered with silver nanoparticles, and the average size in wide range $20-40 \mathrm{~nm}$ with spherical shape, meanwhile for RG the average size in wide range $10-20 \mathrm{~nm}$ in spherical shape too. 


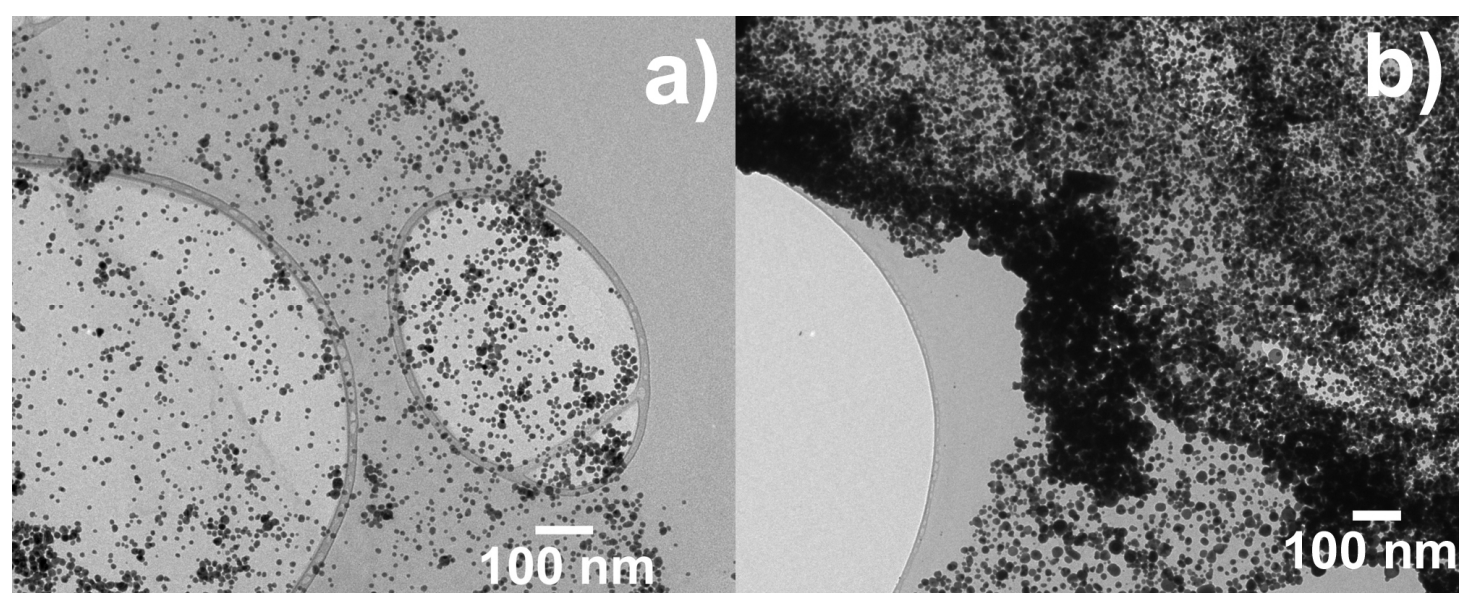

196 197

198

199

200
Figure 7. (a) TEM of RG with Ag NP functionalization; (b) TEM of NDG with Ag nanoparticles functionalization; both samples were conducted under the same experimental conditions.

The average length of silver nanowires obtained in the surface of NDG was a 1 to $2 \mu \mathrm{m}$ and $20-50 \mathrm{~nm}$, it means that the aspect ratio wide/large is in the range of 20-50, SEM micrograph see Figure 8a, TEM micrographs see Figure $8 \mathrm{~b}$ to Figure 8e.
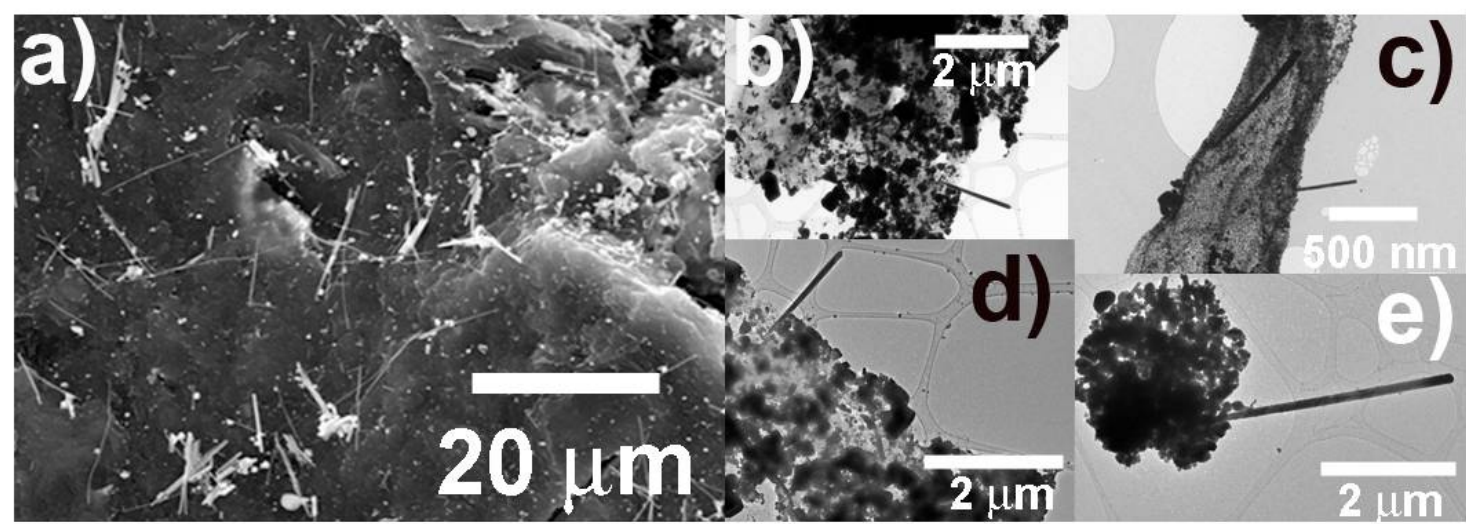

Figure 8. (a) SEM shows silver nanowires in the surface of GN; (b), (c), (d) y (e) TEM of silver nanowires anchored to NDG surface.

The STEM image with an elemental mapping (EDX), shows in the yellow frame: silver nanowires and nanoparticles distribution; in the blue frame: carbon distribution, and nitrogen distribution is in the red one. Figure 9. 


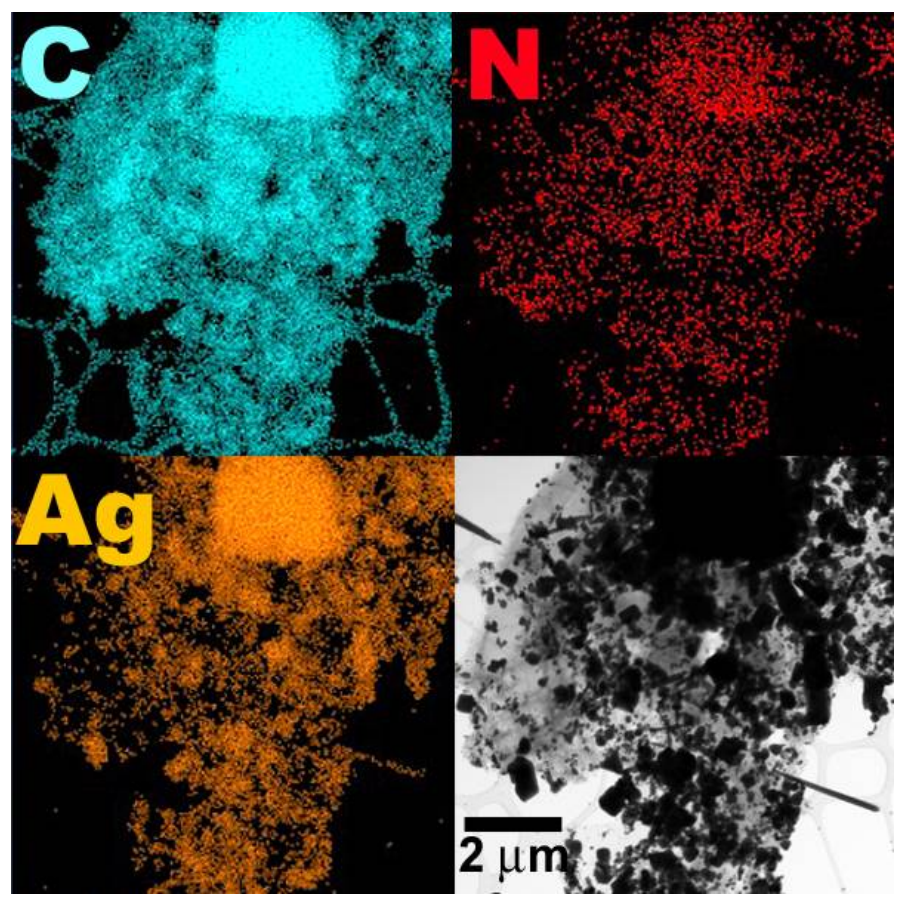

Figure 9. EDX elemental mapping of NDG decorated with Ag nanowires and nanoparticles distribution.

\section{Conclusions}

It is demonstrated that this new proposal experimental method for the synthesis of nitrogen doped Graphene, carried out using caffeine, and its nanowires functionalization it is a useful, easy and green method; We found that the surface of NDG is more reactive due to the content of Nitrogen, and promotes the nucleation of silver nanowires and nanoparticles in the active sites in the surface, and according to these results, it is possibility to used the new method to produce NDG or NW to make Graphene electrodes or circuits interconnected by silver nanowires and used in electronics applications. Analysis, DRG, JJCR and AGR; XPS measurements, HT; Discussion AZO, JJCR and IGE; Supervision, AZO. All authors were involved in the writing process of the manuscript.

Acknowledgments: Thanks to CONACYT for grant 284349. Special thanks to Posgrado CU-Valles UDG, A. Mata Munguía at CUCEI-UDG, M.C. C. G. Elías, M.C. R.L. Tovar, M.C. M. Campos y F.J. Rodríguez (IM. UASLP), Dr. J. M. Romo Herrera (CNYN UNAM), Dra. M. Quintana (CCIASB UASLP), (IPN), Dr. Vicente Garibay F. IMP, and R. Tovar Hurtado IDNANO.

225 Conflicts of Interest: The authors declare no conflict of interest.

\section{References}

1. A. K. Geim and K. S. Novoselov, “The rise of graphene,” Nat. Mater., vol. 6, no. 3, pp. 183-191, 2007.

2. Z. S. Wu, W. C. Ren, L. B. Gao, J. P. Zhao, Z. P. Chen, B. L. Liu, D. M. Tang, B. Yu, C. B. Jiang, and H. M. Cheng, "Synthesis of Graphene Sheets with High Electrical Conductivity and Good Thermal Stability by Hydrogen Arc Discharge Exfoliation94," ACS Nano, vol. 3, no. 2, pp. 411-417, 2009.

3. H. J. Shin, K. K. Kim, A. Benayad, S. M. Yoon, H. K. Park, I. S. Jung, M. H. Jin, H. K. Jeong, J. M. Kim, J. Y. Choi, and Y. H. Lee, "Efficient reduction of graphite oxide by sodium borohydride and its effect on electrical conductance," Adv. Funct. Mater., vol. 19, no. 12, pp. 1987-1992, 2009.

4. A. H. Castro Neto, F. Guinea, N. M. R. Peres, K. S. Novoselov, and A. K. Geim, “The electronic properties of graphene," Rev. Mod. Phys., vol. 81, no. 1, pp. 109-162, 2009. 
5. K. S. Novoselov, Z. Jiang, Y. Zhang, S. V. Morozov, H. L. Stormer, U. Zeitler, J. C. Maan, G. S. Boebinger, P. Kim, and A. K. Geim, “Room-temperature quantum hall effect in graphene," Science (80-. )., vol. 315, no. 5817, p. 1379, 2007.

6. K. I. Bolotin, F. Ghahari, M. D. Shulman, H. L. Stormer, and P. Kim, "Observation of the fractional quantum Hall effect in graphene," Nature, vol. 462, no. 7270, pp. 196-199, 2009.

7. C. L. Kane and E. J. Mele, “Quantum Spin hall effect in graphene,” Phys. Rev. Lett., vol. 95, no. $22,2005$.

8. W. Liu, C. H. Chung, C. Q. Miao, Y. J. Wang, B. Y. Li, L. Y. Ruan, K. Patel, Y. J. Park, J. Woo, and Y. H. Xie, "Chemical vapor deposition of large area few layer graphene on Si catalyzed with nickel films," Thin Solid Films, vol. 518, no. 6 SUPPL. 1, 2010.

9. G. Ning, Z. Fan, G. Wang, J. Gao, W. Qian, and F. Wei, “Gram-scale synthesis of nanomesh graphene with high surface area and its application in supercapacitor electrodes," Chem. Commun., vol. 47, no. 21, p. 5976, 2011.

10. C. N. R. Rao and R. Voggu, "Charge-transfer with graphene and nanotubes," Mater. Today, vol. 13, no. 9, pp. 34-40, 2010.

11. L. Kong, C. Bjelkevig, S. Gaddam, M. Zhou, Y. H. Lee, G. H. Han, H. K. Jeong, N. Wu, Z. Zhang, J. Xiao, P. A. Dowben, and J. A. Kelber, "Graphene/substrate charge transfer characterized by inverse photoelectron spectroscopy," J. Phys. Chem. C, vol. 114, no. 49, pp. 21618-21624, 2010.

12. F. Schedin, A. K. Geim, S. V. Morozov, E. W. Hill, P. Blake, M. I. Katsnelson, and K. S. Novoselov, "Detection of individual gas molecules adsorbed on graphene," Nat. Mater., vol. 6, no. 9, pp. 652-655, 2007.

13. P. Lazar, F. Karlick??, P. Jurecka, M. Kocman, E. Otyepkov??, K. ??af????ov??, and M. Otyepka, “Adsorption of small organic molecules on graphene," J. Am. Chem. Soc., vol. 135, no. 16, pp. 6372-6377, 2013.

14. Q. Su, S. Pang, V. Alijani, C. Li, X. Feng, and K. Müllen, “Composites of Graphene with Large Aromatic Molecules," Adv. Mater., vol. 21, no. 31, pp. 3191-3195, 2009.

15. A. a Balandin, S. Ghosh, W. Bao, I. Calizo, D. Teweldebrhan, F. Miao, and C. N. Lau, "Superior Thermal Conductivity of Single-Layer Graphene 2008," Nano Lett., vol. 8, pp. 902-907, 2008.

16. E. Pop, V. Varshney, and A. K. Roy, "Thermal properties of graphene: Fundamentals and applications," MRS Bull., vol. 37, no. 12, pp. 1273-1281, 2012.

17. M. M. Sadeghi, M. T. Pettes, and L. Shi, "Thermal transport in graphene," Solid State Commun., vol. 152, no. 15, pp. 1321-1330, 2012.

18. S. E. Zhu, S. Yuan, and G. C. A. M. Janssen, “Optical transmittance of multilayer graphene," EPL, vol. 108, no. 1, 2014.

19. C. Synthesis, "Synthesis to TiO 2 Colloids Using Acetic Acid ( Colloidal Suspension for obtaining transparent mesoscopic TiO 2 films )," Glass, pp. 4-6.

20. A. Leela, M. Reddy, A. Srivastava, S. R. Gowda, H. Gullapalli, M. Dubey, and P. M. Ajayan, “Synthesis Of Nitrogen-Doped Graphene," ACS Nano, vol. 4, no. 11, pp. 6337-6342, 2010.

21. Z.-H. Sheng, L. Shao, J.-J. Chen, W.-J. Bao, F.-B. Wang, and X.-H. Xia, “Catalyst-Free Synthesis of NitrogenDoped Graphene via Thermal Annealing Graphite Oxide with Melamine and Its Excellent Electrocatalysis.," ACS Nano., vol. 5, no. 6, pp. 4350-4358, 2011.

22. Y. Shao, S. Zhang, M. H. Engelhard, G. Li, G. Shao, Y. Wang, J. Liu, I. A. Aksay, and Y. Lin, “Nitrogen-doped graphene and its electrochemical applications," J. Mater. Chem., vol. 20, no. 35, p. 7491, 2010.

23. G. Jo, J. Sanetuntikul, and S. Shanmugam, "Boron and phosphorous-doped graphene as a metal-free electrocatalyst for the oxygen reduction reaction in alkaline medium," RSC Adv., vol. 5, no. 66, pp. 53637-53643, 2015.

24. H. Lin, L. Chu, X. Wang, Z. Yao, F. Liu, Y. Ai, X. Zhuang, and S. Han, “Boron, nitrogen, and phosphorous ternary doped graphene aerogel with hierarchically porous structures as highly efficient electrocatalysts for oxygen reduction reaction," New J. Chem., vol. 40, no. 7, pp. 6022-6029, 2016.

25. M. Audiffred, A. L. Elías, H. R. Gutiérrez, F. López-Urías, H. Terrones, G. Merino, and M. Terrones., “Nitrogen-silicon heterodoping of carbon nanotubes," J. Phys. Chem. C, vol. 117, no. 16, pp. 8481-8490, 2013. 
26. A. Zamudio, A. L. Elías, J. A. Rodríguez-Manzo, F. López-Urías, G. Rodríguez-Gattorno, F. Lupo, M. Rühle, D. J. Smith, H. Terrones, D. Díaz, and M. Terrones, “Efficient anchoring of silver nanoparticles on n-doped carbon nanotubes," Small, vol. 2, no. 3, pp. 346-350, 2006.

27. Z. Luo, S. Lim, Z. Tian, J. Shang, L. Lai, B. MacDonald, C. Fu, Z. Shen, T. Yu, and J. Lin, “Pyridinic N doped graphene: synthesis, electronic structure, and electrocatalytic property," J. Mater. Chem., vol. 21, no. 22, p. 8038, 2011.

28. B. P. Vinayan, K. Sethupathi, and S. Ramaprabhu, "Facile synthesis of triangular shaped palladium nanoparticles decorated nitrogen doped graphene and their catalytic study for renewable energy applications," Int. J. Hydrogen Energy, vol. 38, no. 5, pp. 2240-2250, 2013.

29. S. Pearton, “Doped nanostructures.," Nanoscale, vol. 2, no. 7, p. 1057, 2010.

30. D. Geng, S. Yang, Y. Zhang, J. Yang, J. Liu, R. Li, T. K. Sham, X. Sun, S. Ye, and S. Knights, “Nitrogen doping effects on the structure of graphene," Appl. Surf. Sci., vol. 257, no. 21, pp. 9193-9198, 2011.

31. Y. Wang, Y. Shao, D. W. Matson, J. Li, and Y. Lin, "Nitrogen-Doped Graphene and Its Biosensing," ACS Nano, vol. 4, no. 4, pp. 1790-1798, 2010.

32. H. B. Wang, T. Maiyalagan, and X. Wang, "Review on Recent Progress in Nitrogen-Doped Graphene: Synthesis, Characterization, and Its Potential Applications," Acs Catal., vol. 2, no. 5, pp. 781-794, 2012.

33. Y. Wang, Y. Shao, D. W. Matson, J. Li, and Y. Lin, "Nitrogen-doped graphene and its application in electrochemical biosensing," ACS Nano, vol. 4, no. 4, pp. 1790-1798, 2010.

34. R. Lv, Q. Li, A. R. Botello-Méndez, T. Hayashi, B. Wang, A. Berkdemir, Q. Hao, A. L. Elías, R. Cruz-Silva, H. R. Gutiérrez, Y. A. Kim, H. Muramatsu, J. Zhu, M. Endo, H. Terrones, J.-C. Charlier, M. Pan, and M. Terrones, "Nitrogen-doped graphene: beyond single substitution and enhanced molecular sensing," Sci. Rep., vol. 2, pp. 1-8, 2012.

35. X. Li, H. Wang, J. T. Robinson, and H. Sanchez, "Simultaneous Nitrogen-Doping and Reduction of Graphene Oxide Xiaolin Li, Hailiang Wang, Joshua T. Robinson, Hernan Sanchez, Georgi Diankov, Hongjie Dai*," pp. 1-23.

36. D. Marcano, D. Kosynkin, and J. Berlin, “Improved synthesis of graphene oxide," Acs ..., vol. 4, no. 8, pp. 4806-14, 2010.

37. K. K. Caswell, C. M. Bender, and C. J. Murphy, "Seedless, surfactantless wet chemical synthesis of silver nanowires," Nano Lett., vol. 3, no. 5, pp. 667-669, 2003.

38. C. N. R. Rao, K. Gopalakrishnan, and A. Govindaraj, "Synthesis, properties and applications of graphene doped with boron, nitrogen and other elements," Nano Today, vol. 9, no. 3, pp. 324-343, 2014.

39. B. Jiang, S. Song, J. Wang, Y. Xie, W. Chu, H. Li, H. Xu, C. Tian, and H. Fu, “Nitrogen-doped graphene supported Pd@PdO core-shell clusters for C-C coupling reactions," Nano Res., vol. 7, no. 9, pp. 1280-1290, 2014. 